\title{
Hábitos De Consumo De Los Productos Ecológicos En La Provincia De Lugo (España)
}

Cosumption habits of organic products in Lugo province (Spain)

Ibán Vázquez González ${ }^{1}$, Victor Argul Saavedra² María Belén Díaz Hernández $^{3}$

DOI: https://doi.org/10.52719/bjas.v2i2.3754

\section{RESUMEN}

Vista la relevancia territorial de la producción ecológica en España y el contexto caracterizado por un fuerte aumento en la demanda interna de productos ecológicos, este trabajo tiene por objetivo estudiar en un ámbito local (ciudad de Lugo), los hábitos de consumo hacia los productos ecológicos. Se realizaron 177 encuestas personales directas a finales de 2019 a consumidores en tres tipos de establecimientos comerciales, mediante un muestreo aleatorio estratificado por sexo y edad de población. Los resultados confirman un gran conocimiento de lo que son los productos ecológicos, cuyo consumo se produce en 4 de cada 5. La mayor frecuencia de consumo es una vez por semana, siendo las frutas y verduras el producto más consumido. Factores como la edad, nivel de ingresos, formación, hábitos de vida se correlacionan con el consumo. El origen local del producto es el atributo al que el consumidor presta mayor atención y existe una predisposición a pagar un sobreprecio por un producto ecológico, de media del $44 \%$.

Palabras clave: Ecológico. Hábitos consumo. Encuesta consumidores. Disposición al pago. Experimento de elección.

\begin{abstract}
\footnotetext{
${ }^{1}$ Universidade de Santiago de Compostela

${ }^{2}$ Universidade de Santiago de Compostela

${ }^{3}$ Universidade de Santiago de Compostela
}

Acording to the territorial relevance of organic production in Spain and in a context characterized by a strong increase in domestic demand for organic products, the aim of this work is to study in a local scope, the consumption habits towards organic products. For this purpose, 177 personal and direct surveys were realized at the end of 2019 to consumers in three types of commercial establishments, through a random and stratified sampling of population by sex and age. The results confirm a high knowledge about organic products are, being consumed 
in 4 of every 5 interviewed. The highest frequency of consumption is one time per week, being fruits and vegetables the most consumed product. Some factors like age, level of revenues, education, and lifestyles were correlated with a greater consumption. The local origin of the product is the attribute that consumer pays more attention and exist a predisposition to pay an extra price for an organic product, on average a $44 \%$.

Keywords: Organic. Consumption habits. Consumer survey. Willingness to pay. Choice experiment.

\section{INTRODUCCIÓN}

La Unión Europea (Reglamento UE 2018/848) considera la producción ecológica como un sistema general de gestión agrícola y producción de alimentos que combina las mejores prácticas en el medio ambiente y el clima, un alto nivel de biodiversidad, la conservación de los recursos naturales y la aplicación de estándares exigentes de bienestar animal y producción en base a sustancias y procesos naturales. El empleo del término de agricultura ecológica, comienza a emplearse a comienzos del siglo pasado. Howart e Cie (1943), pusieron de manifiesto la correlación entre fertilización mineral, calidad de los alimentos y salud de las personas. Otros trabajos de la época fueron pioneros en el empleo del término "Agricultura ecológica", como Look to the Land escrita en 1940 por Lord Norhbourne's y que enfrentaba la agricultura tradicional frente a la que empleaba productos químicos.

La agricultura ecológica mundial, así como la europea y española, está experimentando un crecimiento sostenido, aunque más pronunciado en la última década. Se caracteriza por un aumento de la superficie y también en el número de agricultores (Willer \& Lernoud, 2019). Esto es atribuible, entre otros muchos factores, a los cambios en los hábitos de consumo de los consumidores y los nuevos estilos de vida más saludables (Hughner, McDonagh, Prothero, Shultz, \& Stanton, 2007; Olaizola, Bernués, Blasco, \& Sanz, 2012).

España es el cuarto país del mundo con la mayor superficie dedicada a la agricultura ecológica (Willer \& Lernoud, 2019), con aproximadamente 2,2 millones de ha y 44.282 operadores en 2018. Tanto la superficie como el número de operadores se ha incrementado de manera significativa desde el año 2008, un 70,5\% y un 86,5\% respectivamente (Ministerio de Agricultura, Pesca y Alimentación [MAPA], 2020). A pesar de que España es el principal país productor europeo, tiene un marcado carácter exportador (Schmid, De Fontguyon, \& Sans, 2007), pues el gasto per cápita de los consumidores españoles, con alrededor de $42,2 €$ en el 

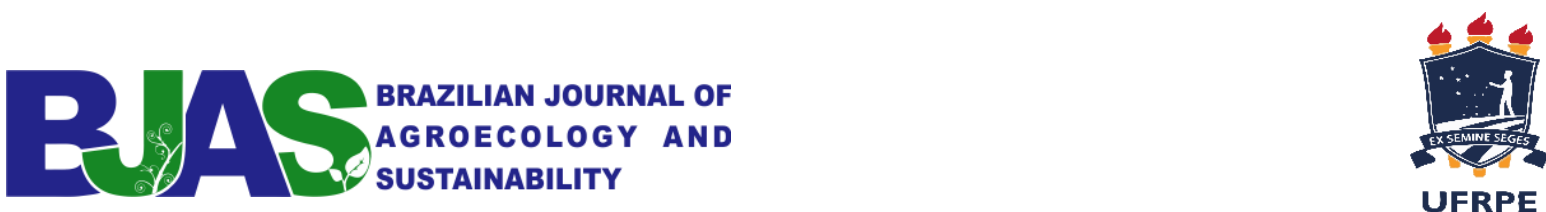

año 2017, es inferior a los 67,2€ de media en la Unión Europea y muy lejos del de otros países del entorno como Suiza (288 €), Dinamarca (278€) y Suecia (237€) (Willer \& Lernoud, 2019; MAPA, 2020). A pesar de que en los últimos años se constata un fuerte incremento del consumo interno, actualmente el consumo se encuentra concentrado en un número reducido de países industrializados (Gonzálvez, 2012).

A nivel de comunidades autónomas, Galicia se sitúa en el 10 puesto en el ranking estatal de superficie (1,5\% del total). Sin embargo, según datos del Consejo Regulador de la Agricultura Ecológica de Galicia (CRAEGA), los 1152 operadores que hay (2,6\% del total de España), tienen una gran importancia, pues es el tercer sello de calidad en facturación, por detrás de la Ternera Gallega y la denominación de origen Rías Baixas. Además, la provincia de Lugo es un referente pues concentra casi la mitad de la superficie ecológica certificada y el $60 \%$ de las explotaciones ganaderas ecológicas.

Vista la relevancia territorial de la producción ecológica en España y el contexto caracterizado por un fuerte aumento en la demanda interna de productos ecológicos, este trabajo tiene por objetivo estudiar en un ámbito local, los hábitos de consumo hacia los productos ecológicos de los consumidores de la ciudad de Lugo. Con ello se tratará de identificar aquellas variables que afectan al consumo, conocer la disposición al pago y otros factores que influyen en la decisión de compra; de tal manera que se pueda obtener una mejor caracterización de los consumidores de productos ecológicos de Lugo.

\section{MATERIAL Y MÉTODOS}

El material empleado procede de una encuesta a 177 consumidores seleccionados entre un total de 82651 habitantes mayores de 18 años que tenía la ciudad de Lugo en el año 2018 (Instituto Galego de Estatística [IGE], 2020a). El tamaño de la muestra se determinó mediante un muestreo aleatorio estratificado para poblaciones finitas, con un nivel de confianza del $95 \%$ y un error de muestreo del 7,5\% (Cea D'Ancona, 2004) (Ecuación 1).

Ecuación 1. Determinación del tamaño muestral.

$$
n=\frac{k^{2} P Q N}{e^{2}(N-1)+k^{2} P Q}
$$

Fuente: Cea D`Ancona (2004) 


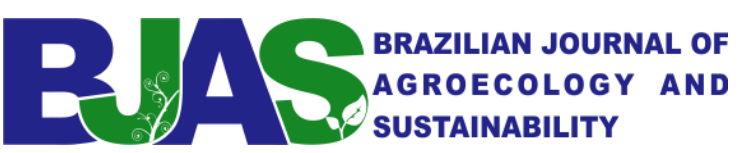

$\mathrm{n}=$ Tamaño necesario de muestra. $\mathrm{N}=$ Tamaño de la población (82651). $\mathrm{K}=$ Coeficiente asociado al nivel de confianza (2). $\mathrm{P}=$ Porcentaje de individuos que consumen productos ecológicos $(0,5) . \mathrm{Q}=$ Porcentaje de individuos que no consumen productos ecológicos $(0,5)$. e= Error de la muestra $(0,075)$.

Las entrevistas se realizaron entre noviembre y diciembre de 2019, de manera aleatoria y proporcional para seis estratos de población en función del sexo y la edad, repartida en tres tipos de establecimientos (supermercado, hipermercado y tienda especializada) (Tabla 1).

Tabla 1. Reparto de la muestra por estrato y establecimiento.

\begin{tabular}{lllllll}
\hline $\begin{array}{l}\text { Estrato (edad- } \\
\text { sexo) }\end{array}$ & $\begin{array}{l}\text { Población } \\
2018>=18 \text { años }\end{array}$ & $\begin{array}{l}\text { Lugo } \\
\text { estrato }\end{array}$ & $\begin{array}{l}\text { Proporción } \\
\text { muestra }\end{array}$ & $\begin{array}{l}\text { Muestra } \\
\text { Supermercado }\end{array}$ & $\begin{array}{l}\text { Muestra } \\
\text { Hipermercado }\end{array}$ & $\begin{array}{l}\text { Muestra tienda } \\
\text { especializada }\end{array}$ \\
\hline 18-34Hombre & 8058 & 9,7 & 18 & 6 & 6 & 6 \\
18-34Mujer & 8294 & 10,0 & 18 & 6 & 6 & 6 \\
35-64Hombre & 21047 & 25,5 & 45 & 15 & 15 & 15 \\
35-64Mujer & 24294 & 29,4 & 51 & 17 & 17 & 17 \\
$>=65$ Hombre & 8521 & 10,3 & 18 & 6 & 6 & 6 \\
$>=65$ Mujer & 12437 & 15,0 & 27 & 9 & 9 & 9 \\
\hline Total & 82651 & 100,0 & 177 & 59 & 59 & 59 \\
\hline
\end{tabular}

Fuente: Elaboración propia

La encuesta, que tuvo una duración media de 15 minutos, constaba de un total de 38 preguntas, estructuradas en 5 secciones: 1-Conocimiento de productos ecológicos; 2-Consumo de productos ecológicos; 3-Factores que influyen en la decisión de compra; 4-Valoración del producto; 5-Perfil del entrevistado. En el bloque 4, relacionado con la valoración del producto, se realizó un experimento de elección para tres productos al azar (leche UHT, manzana y un filete de ternera $1^{\circ} \mathrm{A}$ ), planteando 5 escenarios con tres opciones posibles (A, B y C (NS-NC)), resultado de la combinación de atributos como el producto (ecológico-convencional), origen (Galicia-fuera) y precio (alto y bajo). También se hicieron preguntas abiertas para conocer la máxima disposición al pago para los tres productos anteriores, en ecológico y convencional.

Para el tratamiento estadístico de los datos se emplearon diferentes análisis de estadísticos descriptivos (frecuencias, valores medios), de varianza (ANOVA) y de relaciones (Chi cuadrado), según estrato de muestreo. El programa informático utilizado ha sido IBM SPSS (versión 22). 


\subsection{Perfil del entrevistado}

El nivel de estudios de la población entrevistada es relativamente alto, pues algo más de la mitad tiene estudios superiores (36,7\% universitarios y 15,3\% FP2); esto podría venir condicionado por la proximidad de los establecimientos escogidos al campus universitario de Lugo. La principal ocupación de los entrevistados es trabajo por cuenta ajena (33,9\%); le siguen un $25,4 \%$ de jubilados y un $15,8 \%$ de funcionarios; en situación de desempleo estaban un 7,9\% de los entrevistados.

En relación con la composición de la familia (número de miembros), un mayor porcentaje de hogares entrevistados $(30,5 \%)$ lo componen 3 miembros, si bien la mayoría $(85,3 \%)$ están compuestos por entre 2 y 4 miembros. En relación con el nivel de ingresos mensual de la unidad familiar, predominan los hogares con entre 1000 y $2000 €(36,7 \%)$; sin embargo, nos llama la atención que un 56,5\% de los entrevistados tiene un nivel de ingresos superior $(27,7 \%$ de 2000 a menos de $3000 €$ y un $28,8 \%$ mayor o igual a $3000 €)$, al ser Lugo una de las capitales de provincia con menor renta per cápita (IGE, 2020b). Esto puede guardar relación con el elevado porcentaje de entrevistados que posee estudios superiores, o la proximidad de los establecimientos al campus universitario.

La mayor parte de los entrevistados afirma tener un estilo de vida saludable, controlando la ingesta de sal, realizando ejercicio físico con regularidad, comiendo con frecuencia frutas y verduras o revisando periódicamente la salud. Un sorprendente $10 \%$ afirma comprar alimentos por internet, fundamentalmente aquellos con entre 35 y 64 años; este porcentaje es muy superior a las cifras que se desprenden del último informe de Consumo alimentario en España (1,3\% del volumen) (MAPA, 2019).

\subsection{Conocimientos sobre productos ecológicos}

El $96 \%$ de los consumidores afirman saber lo que significa que un producto sea de origen ecológico. En comparación con un trabajo similar realizado 10 años antes en la misma ciudad de Lugo (Oviedo, 2009), había un 88\% de los entrevistados lo sabían, por lo que se constata un incremento del grado de conocimiento con el tiempo. En los dos grupos formados 
por hombres y mujeres jóvenes, entre 18 y 34 años, existe un mayor conocimiento, que se corresponde con los resultados de otros trabajos (León \& Martínez, 2011).

El conocimiento real que los entrevistados tienen sobre los productos ecológicos es algo inferior al que manifiestan tener. Así, un 74,6\% de los entrevistados reconoce el sello identificativo del CRAEGA y un $71 \%$ reconoce los sellos nacionales o europeos de productos ecológicos entre una lista de 30 sellos relacionados con productos sostenibles. Existen diferencias significativas al nivel del $5 \%$ en el reconocimiento del sello del CRAEGA según perfil del entrevistado, habiendo un menor grado de conocimiento entre los mayores, especialmente mujeres.

La práctica totalidad de los entrevistados $(92,6 \%)$ concibe un producto ecológico como un alimento sin aditivos químicos $(62,1 \%)$ o más sano $(32,1 \%)$. Esto coincide con lo encontrado en otros trabajos (Fuentes \& López, 2008; Dupupet, Valor, \& Labajo, 2010) y da muestra del elevado grado de conocimiento que manifiestan o realmente saben sobre los productos ecológicos.

Tabla 2. Conocimiento sobre productos ecológicos (\% sobre total y valor medio), según perfil del entrevistado.

\begin{tabular}{|c|c|c|c|c|c|c|c|}
\hline & $18-$ & $18-$ & $35-$ & $35-$ & $>=65$ & $>=65$ & Tota \\
\hline \% SOBRE EL TOTAL DE ENTREVISTADOS & $34 \mathrm{H}$ & $34 \mathrm{M}$ & $64 \mathrm{H}$ & $64 \mathrm{M}$ & $\mathrm{H}$ & M & 1 \\
\hline Afirma conocer lo que es un p. ecológico ${ }^{\text {ns }}$ & 100,0 & 100,0 & 97,8 & 98,0 & 94,4 & 85,2 & 96,0 \\
\hline Reconoce distintivo p. ecológico CRAEGA*** & 77,8 & 100,0 & 77,8 & 82,4 & 61,1 & 44,4 & 74,6 \\
\hline Selecciona distintivo correcto p. ecológico ${ }^{\text {ns }}$ & 78,0 & 78,0 & 76,0 & 75,0 & 61,0 & 51,0 & 71,0 \\
\hline Afirma un p. ecológico es más sano ${ }^{\text {ns }}$ & 27,8 & 22,2 & 28,9 & 25,5 & 44,4 & 40,7 & 30,5 \\
\hline Afirma un p. ecológico es sin aditivos químicos ${ }^{\mathrm{ns}}$ & 66,7 & 66,7 & 64,4 & 70,6 & 44,4 & 48,1 & 62,1 \\
\hline Precio principal problemática p. ecológico* & 66,6 & 94,4 & 84,4 & 86,3 & 61,1 & 88,9 & 82,4 \\
\hline \multicolumn{8}{|l|}{ Valor medio } \\
\hline Años escuchan concepto p. ecológico* & 6,3 & 6,8 & 10,8 & 10,6 & 8,9 & 8,3 & 9,3 \\
\hline
\end{tabular}

Fuente: elaboración propia.

Significación estadística: ${ }^{*}(5 \%), * *(1 \%), * * *(0,1 \%)$, ns (no)

La principal problemática o debilidad de los productos ecológicos para un $82 \%$ de los encuestados es que son productos más caros. Existen diferencias significativas al nivel del 5\% según perfil de entrevistado, considerando menos problema el precio los hombres jóvenes y mayores. Muchos autores coinciden y han asociado el precio como el principal limitante en la 


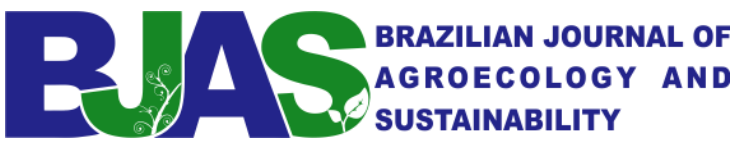

compra de productos ecológicos (Albardíaz, 1998; Gracia, Gil, \& Sánchez, 1999; Gil, Gracia, \& Sanchez, 2000; Mesías, Martínez-Carrasco, Martínez, \& Gaspar, 2011).

A la hora de cuantificar el espacio de tiempo que los entrevistados escuchan hablar del concepto ecológico este ha sido de 9,3 años. Teniendo en cuenta que los productos ecológicos tienen presencia en el mercado agroalimentario español desde hace más de 20 años, hace pensar en la lenta velocidad de propagación en el ámbito local de este concepto. A nivel de estrato se observan diferencias significativas ( $\mathrm{p}$-valor $<0,05$ ), siendo los jóvenes los que menos tiempo han escuchado este concepto.

\subsection{Consumo de productos ecológicos}

La mayor parte de la población entrevistada (81\%) afirma consumir o haber consumido productos ecológicos en su hogar, siendo los más consumidos las frutas o las verduras (41\%). Oviedo (2009), determina que un 58\% de la población consumía o había consumido alimentos ecológicos en Lugo 10 años antes, lo que confirma el fuerte incremento en el consumo de productos ecológicos en los últimos años (MAPA, 2020). Además, el mayor consumo de frutas y verduras puede estar relacionado con múltiples factores como su asociación hacia producto ecológico (Fuentes \& López, 2008), o la concepción ambiental beneficiosa de este tipo de productos (Shrum \& McCarty, 1995). Según estrato de población, existe una tendencia significativa $(0,05<\mathrm{p}$-valor $<0,1)$ en relación al consumo, siendo menor en los entrevistados con mayor edad.

Tabla 3. Consumo de productos ecológicos (\% sobre total y número de entrevistados), según perfil del entrevistado.

\begin{tabular}{|c|c|c|c|c|c|c|c|}
\hline \% SOBRE EL TOTAL DE ENTREVISTADOS & $18-34 \mathrm{H}$ & $18-34 \mathrm{M}$ & $35-64 \mathrm{H}$ & $35-64 \mathrm{M}$ & $>=65 \mathrm{H}$ & $>=65 \mathrm{M}$ & Total \\
\hline Afirma haber consumido p.ecológicos ${ }^{t}$ & 82,5 & 66,5 & 88,0 & 91,0 & 72,0 & 66,5 & 80,7 \\
\hline Consumen frutas o verduras ${ }^{\mathrm{ns}}$ & 38,9 & 38,9 & 42,2 & 51,0 & 33,3 & 29,6 & 41,2 \\
\hline \multicolumn{8}{|c|}{ \% SOBRE EL TOTAL CONSUMEN P. ECOLÓGICOS (MOTIVO Y FRECUENCIA) } \\
\hline Consumen p.ecológicos por ser más sanos ${ }^{\mathrm{ns}}$ & 33,3 & 33,3 & 46,2 & 50,0 & 61,5 & 44,4 & 46,1 \\
\hline Consumen p.ecológicos por ser beneficiosos m.a. ${ }^{t}$ & 60,0 & 50,0 & 41,0 & 39,1 & 15,8 & 22,2 & 38,4 \\
\hline Consumen p.ecológicos por mejor sabor ${ }^{\mathrm{ns}}$ & 6,6 & 16,6 & 7,7 & 10,9 & 23,1 & 33,3 & 13,9 \\
\hline Consumen P. ecológicos semanalmente ${ }^{\text {ns }}$ & 46,6 & 8,3 & 25,6 & 32,6 & 30,7 & 16,6 & 27,9 \\
\hline \multicolumn{8}{|c|}{ \% SOBRE EL TOTAL QUE NO CONSUME P. ECOLÓGICOS (MOTIVOS) } \\
\hline M1-Producto igual que convencional ${ }^{\text {ns }}$ & 0,0 & 16,6 & 50,0 & 20,0 & 0,0 & 11,1 & 17,6 \\
\hline M2-Demasiado caros ${ }^{* *}$ & 100,0 & 66,6 & 16,66 & 80,0 & 0,0 & 22,2 & 41,2 \\
\hline
\end{tabular}




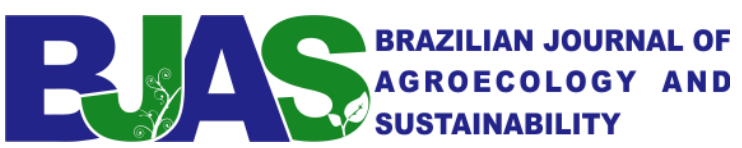

M3-Difícil de encontrar ${ }^{\text {ns }}$

$0,0 \quad 16,6 \quad 33,3 \quad 0,0$

20,0

0,0

UFRPE

M4-Cultivo alimentos *

Fuente: Elaboración propia.

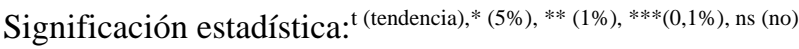

El principal motivo de consumo de productos ecológicos está relacionado con ser un producto más sano ( $46 \%$ de los que consumen), por sus prácticas beneficiosas para el medioambiente (39\%) o su mejor sabor (14\%); vemos por lo tanto como la salubridad se consolida como uno de los principales motivos para su consumo (Sánchez, Etxaniz, \& Tekelioglu, 1997). Existe una tendencia significativa $(0,05<p$-valor $<0,1)$ en relación al motivo medioambiental, destacando una mayor preocupación entre los entrevistados jóvenes al igual que los que acuden a tiendas especializadas.

La mayor frecuencia de consumo de los productos ecológicos es una vez por semana (28\% de los que consumen), seguida por una vez al mes $(22,4 \%)$, cada 3-4 días $(18,9 \%)$, diariamente $(18,9 \%)$ o sólo en ocasiones especiales $(11,9 \%)$.

Entre los 34 entrevistados que no consumen productos ecológicos el principal motivo es que son demasiado caros $(41 \%)$ o que no lo ven necesario porque cultivan sus propios alimentos $(38 \%)$. En ambos existen diferencias significativas en función del estrato de muestreo; así los entrevistados más jóvenes son más sensibles al mayor precio, mientras que los más longevos en mayor medida cultivan sus propios alimentos, considerando que tienen producto de calidad (Fuentes \& López, 2008).

\subsection{Factores que influyen en la decisión de compra}

A la mitad de los entrevistados (50,3\%), le resulta fácil encontrar productos ecológicos, mientras que a un $28,3 \%$ le resulta complicado. Se pone de manifiesto que a pesar del aumento del consumo y del número de referencias, todavía sigue siendo un problema la disponibilidad de productos ecológicos en los lineales (Bonini \& Oppenheim, 2008). Esta cierta dificultad en encontrar productos ecológicos se correlaciona con que la valoración que hacen de la oferta de productos ecológicos en el mercado (5,6 puntos) no es del todo satisfactoria.

La mayor parte de los entrevistados afirman no desconfiar de los productos ecológicos certificados (84\%); sin embargo, hay un $16,4 \%$ que si lo hace lo cual supone un obstáculo para la compra de estos productos (McEachern, Seaman, Padel, \& Foster, 2005). Según estrato de la muestra se asocia una mayor confianza en los entrevistados de menor edad, mientras que el 
principal motivo de desconfianza, para aproximadamente 2 de cada $3(62 \%)$, es que consideran que el producto ecológico es igual que el convencional; por lo que puede estar fallando la transmisión en el propio envase (Carrero \& Valor, 2012). En este sentido un $83 \%$ de los entrevistados que desconfían consideran fundamental un aumento de la transparencia en todo el proceso; si bien pierde relevancia entre los entrevistados de mayor edad.

El aspecto al que el consumidor da mayor relevancia a la hora de comprar un producto ecológico es el origen (Gázquez Abad, Jiménez Guerrero, \& Linares Agüera, 2010), gallego (9 puntos) o español (8,5 puntos), seguido del precio (8,2 puntos). Por su contra los aspectos menos valorados son el aspecto visual del producto (6,5 puntos), el sello de calidad diferenciada (6,6 puntos) o el aspecto del local (6,7 puntos).

Tabla 4. Factores influyen decisión compra (\% sobre total y valor medio), según perfil del entrevistado.

\begin{tabular}{|c|c|c|c|c|c|c|c|}
\hline \% SOBRE EL TOTAL DE ENTREVISTADOS & $18-34 \mathrm{H}$ & $18-34 \mathrm{M}$ & $35-64 \mathrm{H}$ & $35-64 \mathrm{M}$ & $\begin{array}{l}>=65 \\
\mathrm{H}\end{array}$ & $>=65 \mathrm{M}$ & $\begin{array}{l}\text { Tota } \\
1\end{array}$ \\
\hline Consideran fácil encontrar p. ecológicos ${ }^{\mathrm{ns}}$ & 66,7 & 44,4 & 51,1 & 52,9 & 38,9 & 44,4 & 50,3 \\
\hline Desconfía de los p. ecológicos ${ }^{\mathrm{ns}}$ & 11,1 & 5,6 & 17,8 & 17,6 & 22,2 & 18,5 & 16,4 \\
\hline \multicolumn{8}{|c|}{ \% SOBRE EL TOTAL QUE DESCONFÍAN P. ECOLÓGICOS (PRINCIPAL MOTIVO Y MEDIDA) } \\
\hline Motivo - p.ecológico igual convencional ${ }^{\text {ns }}$ & 50,0 & 0,0 & 75,0 & 55,6 & 75,0 & 60,0 & 62,1 \\
\hline Medida - transparencia eliminar desconfianza** & 100,0 & 100,0 & 100,0 & 100,0 & 75,0 & 40,0 & 82,8 \\
\hline \multicolumn{8}{|l|}{ VALOR MEDIO (0 A 10 PUNTOS) } \\
\hline Oferta de p. ecológicos (0 Insuf. y 10 suficiente) $)^{\mathrm{ns}}$ & 5,2 & 5,4 & 5,7 & 5,3 & 5,9 & 5,8 & 5,6 \\
\hline \multicolumn{8}{|c|}{ Grado de importancia (0 Nada importante a 10 muy importante) aspectos compra p.ecológicos } \\
\hline 1-Origen España $\mathrm{a}^{\mathrm{ns}}$ & 8,2 & 8,3 & 8,3 & 8,7 & 8,6 & 8,9 & 8,5 \\
\hline 2-Origen Galicia ${ }^{\mathrm{ns}}$ & 8,7 & 8,5 & 9,0 & 9,1 & 9,0 & 9,3 & 9,0 \\
\hline 3-Información etiqueta ${ }^{\mathrm{ns}}$ & 8,6 & 7,9 & 8,0 & 8,1 & 8,0 & 7,7 & 8,0 \\
\hline 4-Sello de calidad diferenciada ${ }^{\text {ns }}$ & 6,4 & 6,9 & 6,7 & 6,1 & 7,3 & 6,8 & 6,6 \\
\hline 5-Aspecto visual producto ${ }^{\mathrm{ns}}$ & 6,8 & 5,6 & 7,0 & 6,4 & 5,9 & 6,6 & 6,5 \\
\hline 6-Aspecto local compra ${ }^{\text {ns }}$ & 6,7 & 6,2 & 7,0 & 7,1 & 5,9 & 6,4 & 6,7 \\
\hline 7-Asesoramiento vendedor ${ }^{*}$ & 7,2 & 6,6 & 7,6 & 6,8 & 6,9 & 7,0 & 7,1 \\
\hline 8-Precio ${ }^{\text {ns }}$ & 7,9 & 8,4 & 7,8 & 8,1 & 7,9 & 9,0 & 8,2 \\
\hline
\end{tabular}

Fuente: Elaboración propia

Significación estadística: ${ }^{t}$ (tendencia), ${ }^{*}(5 \%), * *(1 \%), * * *(0,1 \%)$, ns (no) 


\subsection{Valoración del producto}

Independientemente del producto planteado (leche, filete o manzana) vemos que la tarjeta elegida (A, B o C) en cada una de las 5 plantillas de elección ha tenido un peso relativo similar. El atributo que ha tenido un mayor poder de decisión a la hora de decantar la balanza por una tarjeta u otra ha sido el origen local (gallego) (Figura 1).

En la primera plantilla la preferencia es un producto ecológico, local y más caro frente a uno convencional, foráneo y más barato. El porcentaje de entrevistados que escogen esta opción varía entre un $66 \%$ en caso del filete y un $81 \%$ en el caso de la manzana. En la segunda plantilla la preferencia es un producto convencional, local y económico frente a uno ecológico, foráneo y más caro ( $68 \%$ en el caso de la manzana y un $70 \%$ en el caso del filete). En la tercera plantilla la preferencia es un producto ecológico, local y económico frente a uno convencional, también local y económico ( $89 \%$ filete y un $96 \%$ manzana); por lo que a igualdad de atributos de origen y precio se escoge un producto ecológico. En la cuarta plantilla la preferencia es un producto ecológico, local y más caro frente a uno ecológico, foráneo y más barato (62\% filete y $75 \%$ leche); por lo que el origen se antepone al precio. En la quinta plantilla reafirma lo anterior, pues tenemos un mismo producto ecológico a un mismo precio y varía el origen, siendo el origen local la preferencia escogida.

Figura 1. Distribución respuestas experimento elección.

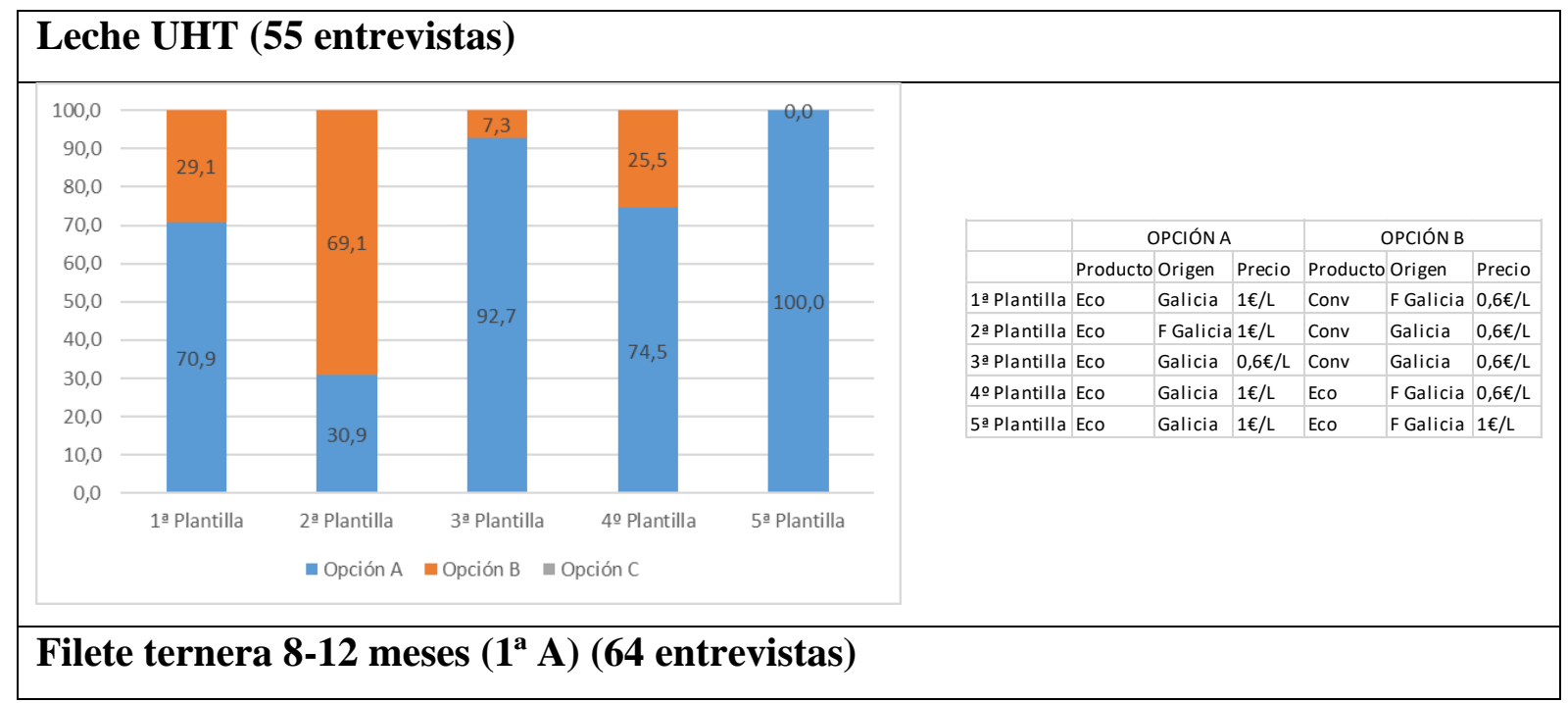




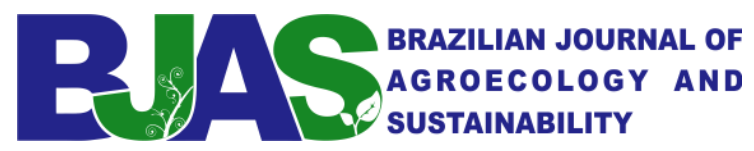

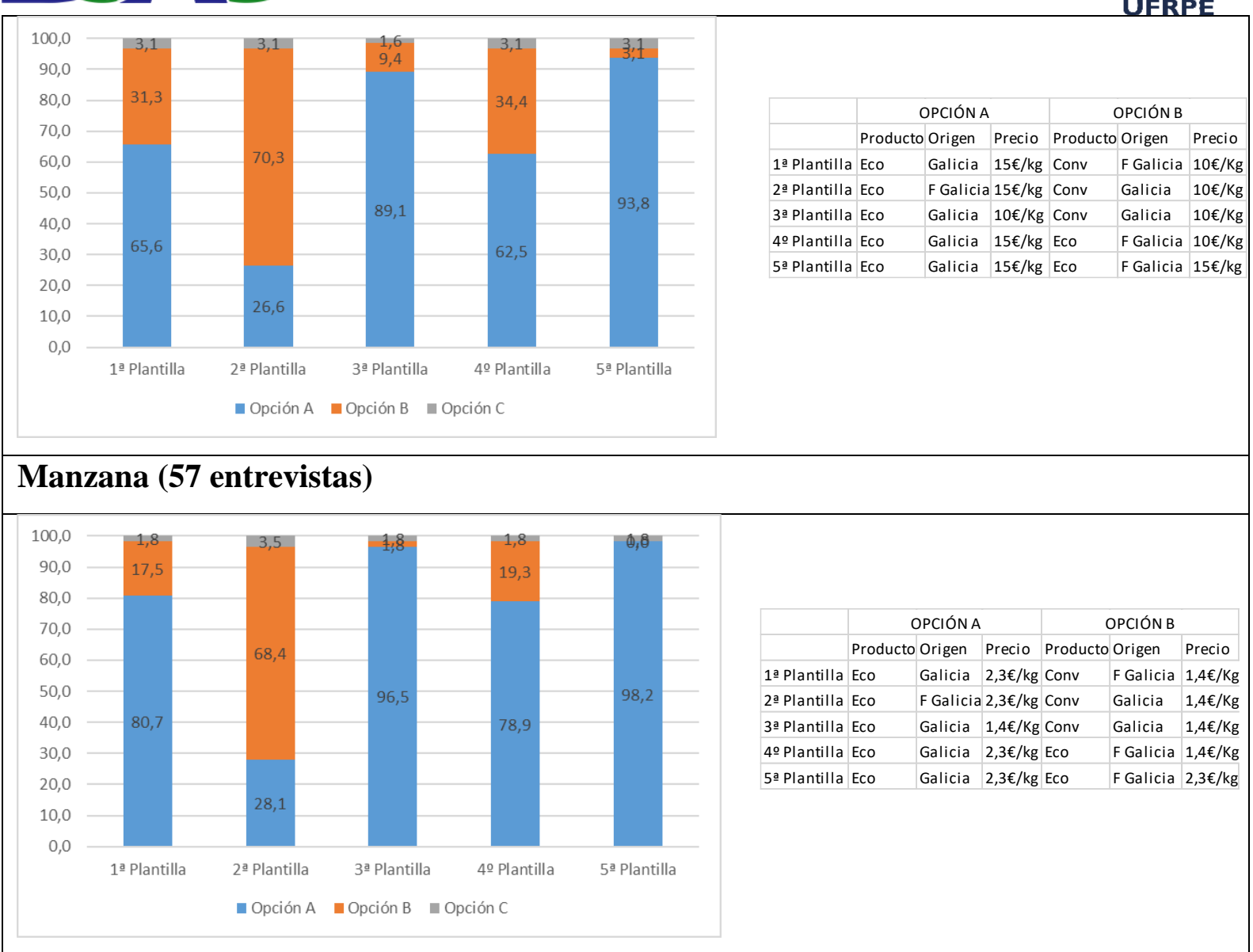

Fuente: Elaboración propia

Prácticamente la totalidad de los entrevistados se han mostrado partidarios de pagar más por un producto ecológico, de media un 44\%. El mayor incremento porcentual en la disposición al pago se encuentra en las manzanas, con el $54 \%$, seguido en la leche con un $46 \%$ y en la carne con un $32 \%$. De esta manera se confirma la mayor disposición al pago de un producto ecológico frente a uno convencional (Díaz, Pleite, Martínez-Paz, \& García, 2011), en unos porcentajes similares al sobreprecio que la mayoría (110) considera que tiene (entre un 25 y 50\%); además se produce lo que apuntan otros autores de una mayor disposición al pago por frutas y verduras (Gil, Gracia, \& Sanchez, 2000).

Tabla 5. Disposición máxima al pago

\begin{tabular}{llllllcc}
\hline PRECIO MÁXIMO DISPOSICIÓN AL PAGO & $\mathbf{1 8 - 3 4 H}$ & $\mathbf{1 8 - 3 4 M}$ & $\mathbf{3 5 - 6 4 H}$ & $\mathbf{3 5 - 6 4 M}$ & $\mathbf{>}=\mathbf{6 5 H}$ & $\mathbf{>}=\mathbf{6 5 M}$ & Total \\
\hline Precio máximo leche convencional $(€ / \mathrm{L})^{\mathrm{ns}}$ & 0,98 & 0,91 & 1,02 & 0,98 & 0,9 & 1,21 & 1,01 \\
Precio máximo filete ternera $1^{\mathrm{a}}$ A convencional $(€ / \mathrm{Kg})^{\mathrm{ns}}$ & 8,27 & 8,13 & 8,64 & 9,11 & 8,18 & 7,46 & 8,46 \\
Precio máximo manzana convencional $(€ / \mathrm{kg})^{\mathrm{ns}}$ & 1,8 & 1,28 & 1,52 & 1,48 & 1,46 & 1,28 & 1,47 \\
\hline Precio máximo leche ecológico $(€ / \mathrm{L})^{\mathrm{ns}}$ & 1,49 & 1,3 & 1,46 & 1,46 & 1,78 & 1,4 & 1,47
\end{tabular}




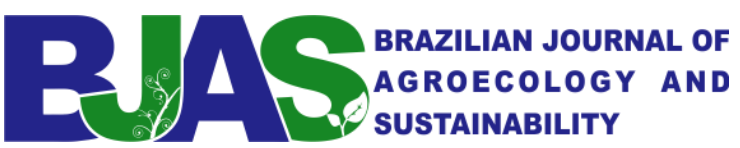

Precio máximo filete ternera $1^{\mathrm{a}} \mathrm{A}$ ecológico $(€ / \mathrm{Kg})^{\mathrm{ns}}$

Precio máximo manzana ecológico $(€ / \mathrm{kg})^{\mathrm{ns}}$

10,89

$11,79 \quad 11,8$

UFRPE

PORCENTAJE DE INCREMENTO SOBREPRECIO ECOLÓGICO

Incremento promedio leche ecológico

$51,8 \quad 43,8$

2,44

2,31

$2,1 \quad 2,13$

Incremento promedio filete ternera ecológico

$23,1 \quad 34,1$

42,6

48,5

97,2

15,6

45,8

Incremento promedio manzana ecológica

$41,7 \quad 42,4$

36,5

29,6

37,6

27,3

32,0

Fuente: Elaboración propia.

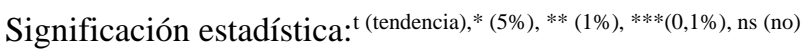

\section{CONCLUSIONES}

Las principales conclusiones de este trabajo son las siguientes:

El perfil de consumidor entrevistado destaca por la alta formación académica, elevados ingresos familiares y un estilo de vida saludable.

La práctica totalidad de los entrevistados afirma saber lo que es un producto ecológico; sin embargo, se constata un grado de conocimiento inferior a la hora de proceder a identificar las etiquetas. Se concibe un producto ecológico como aquel sin aditivos químicos y más sano; además consideran que su principal debilidad es que son más caros.

La media de años que los encuestados han escuchado hablar de los productos ecológicos es relativamente pequeña y hace pensar en la escasa velocidad de transmisión a escala local de este concepto y su potencial de crecimiento.

Cuatro de cada cinco entrevistados afirman haber consumido productos ecológicos. Entre los motivos de consumo destaca la salud o la preocupación por el medio ambiente. La mayor frecuencia de consumo es una vez por semana y el producto consumido son las frutas y las verduras. Se asocia un mayor consumo cuanto menor es la edad, hábitos de vida saludables o compra en establecimientos especializados. A pesar del aumento del consumo y del número de referencias, todavía resulta complicado encontrar productos ecológicos en los lineales.

Una parte significativa de las personas que no consumen productos ecológicos lo hacen por su superior precio y por disponer de producciones propias. Además, hay un porcentaje reducido pero significativo, de consumidores que desconfía de los productos ecológicos, considerando que son iguales que los convencionales y que se debería aumentar su transparencia.

Los atributos a los que el consumidor presta una mayor atención a la hora de comprar productos ecológicos son la procedencia, principalmente gallega, y también el precio. Se 

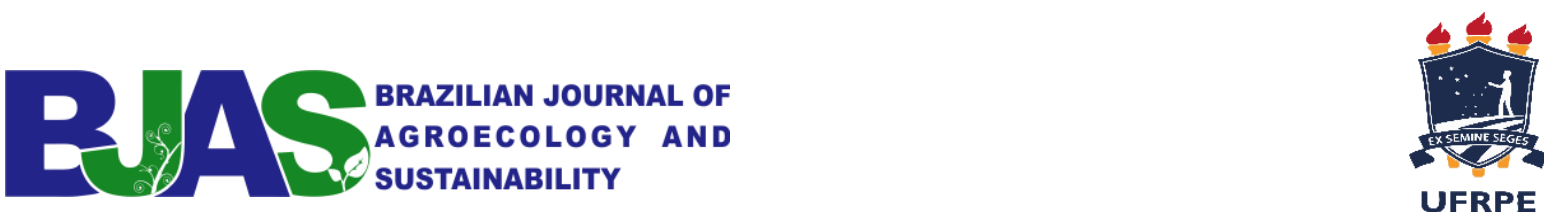

consideran menos relevantes el sello de calidad diferenciada, el aspecto visual del producto o del establecimiento de compra.

En el experimento de elección el origen gallego ha tenido un mayor poder de decisión que el tipo de producto y el precio, no observando diferencias en las elecciones en función del producto.

Para finalizar existe una mayor disposición al pago por productos ecológicos, de media un $44 \%$ más, lo que se sitúa en el umbral de sobreprecios que la mayoría de entrevistados estarían dispuestos a pagar. Además, existen diferencias en función del alimento, en frutas como las manzanas existe una mayor disposición al pago, al igual que en tiendas especializadas.

\section{REFERENCIAS}

Albardiaz, M. A. (1998). Estudio de los frenos al desarrollo de la agricultura ecológica a través de las variables del consumo. Distribución y Consumo, 38, 112-119.

Bonini, S., \& Oppenheim, J. (2008). Cultivating the green consumer. Stanford Social Innovation Review, 6(4), 56-61.

Carrero, I., \& Valor, C. (2012). CSR-labelled products in retailers' assortment. International Journal of Retail \& Distribution Management, 40(8), 629-652.

Cea D'Ancona, M. A. (2004). Métodos de encuestas, teoría y práctica, errores y mejoras.

Díaz, F. M., Pleite, F. M. C., Martínez-Paz, J. M., \& García, P. G. (2011). La disposición a pagar por alimentos ecológicos en España: una aproximación a la existencia de diferencias regionales. ITEA, Información Técnica Económica Agraria, 107(1), 3-20. Recuperado de https://dialnet.unirioja.es/servlet/articulo?codigo $=3583022$.

Dupupet, A., Valor, C., \& Labajo, V. (2010). Comercialización minorista de productos biológicos. Distribución y consumo, 112, 63-63.

Fuentes, C., \& López de Coca, E. (2008). El consumo de productos ecológicos. Distribución y consumo, 99, 5-24.

Gil, J. M., Gracia, A., \& Sanchez, M. (2000). Market segmentation and willingness to pay for organic products in Spain. International Food and Agribusiness Management Review, 3, 207-226.

Gonzálvez, V. (2012). Situación actual y perspectivas de la agricultura ecológica en España. Dossier revista vida rural. 

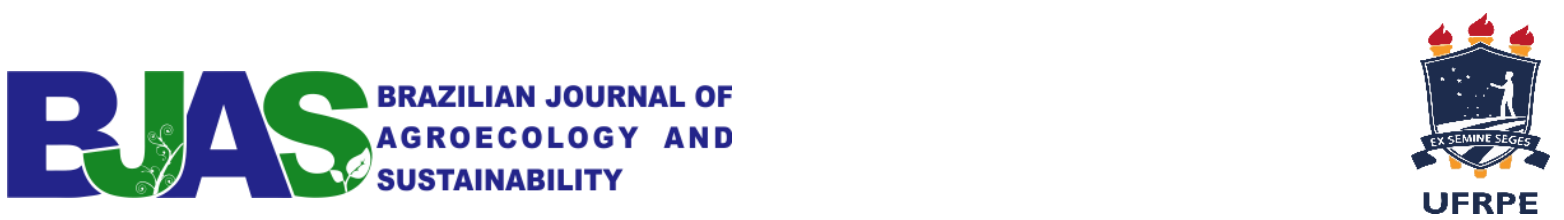

Gracia, A., Gil, J. M., \& Sánchez, M. (1999). Factors affecting consumers willingness to pay for organic food products. IX European Congress of Agricultural Economics, Varsovia, Polonia.

Howard, S. A., \& ClE, M. A. (1943). An agricultural testament. Prabhat Prakashan.

Hughner, R. S, McDonagh, P., Prothero, A., Shultz, C. J., \& Stanton, J. (2007). Who are organic food consumers? A compilation and review of why people purchase organic food. Journal of Consumer Behaviour J. Consumer Behav, 6, 94-110.

Instituto Galego de Estatística. (2020a). Padrón municipal de habitantes. Lugo año 2018.

Instituto Galego de Estatística. (2020b). Renta municipal dos sector fogares. Ano 2017.

Gázquez Abad, J. C., Jiménez Guerrero, J. F., Linares Agüera, E. (2010). La importancia del "país de origen" en la fase de evaluación del consumidor de productos hortícolas. Revista Española de Estudios Agrosociales y Pesqueros, 225, 47-69. Recuperado de https://www.mapa.gob.es/app/publicaciones/art_datos_art.asp?articuloid=1280\&codrevis ta=REEAP.

LANDIS D. A. (2017) Designing agricultural landscapes for biodiversity-based ecosystem services. Basic and Applied Ecology 18:1. doi:10.1016/j.baae.2016.07.005

León, A. T., \& Martínez, J. V. (2011). El consumo verde en México: Conocimiento, actitud y comportamiento. In XVI Congreso Internacional de Contaduría, Administración $e$ Informática. México, ANFECA.

Ministerio de Agricultura, Pesca y Alimentación. (2019). Informe de consumo alimentario en España 2018. Madrid.

Ministerio de Agricultura, Pesca y Alimentación. (2020). Agricultura ecológica, estadísticas 2018. Madrid.

McEachern, M., Seaman, C., Padel, S., \& Foster, C. (2005). Exploring the gap between attitudes and behaviour. British Food Journal, 107(8), 606-625.

Mesías, F. J., Martínez-Carrasco, F., Martínez, J. M., \& Gaspar, P. (2011). Functional and organic eggs as an alternative to conventional production: A conjoint analysis of consumers' preferences. Journal of the Science of Food and Agriculture, 91(3), 532-538.

Olaizola, A., Bernués, A., Blasco, I., \& Sanz, A. (2012). Perspectivas de una carne de calidad diferenciada: análisis exploratorio para la carne de vacuno Serrana de Teruel. ITEA Información Técnica Económica Agraria, 108, 546-562.

Oviedo, M. (2009). Estudio técnico sobre los canales de comercialización de productos ecológicos en la ciudad de Lugo y análisis de alternativas para la mejora de su eficiencia. Universidad de Santiago de Compostela. EPS Lugo. 


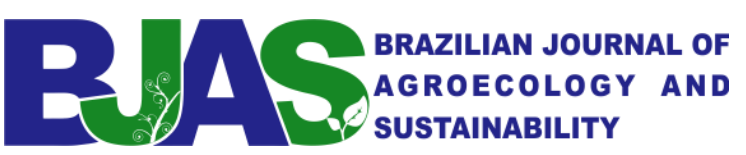

Sánchez, M., Etxaniz, M., \& Tekelioglu, I. (1997). Análisis de las preferencias en el consumo de productos de agricultura ecológica. Estudios sobre consumo, 41, 49-63.

Schmid, O., De Fontguyon, G., \& Sans, P. (2007). Desarrollo del mercado de productos de la agricultura ecológica en Europa: un análisis de sus condiciones y del papel de las iniciativas comerciales. Rev. Esp. Est. Agrosoc. Pesq., 214, 15-44.

Shrum, L. J., \& McCarty, J. A. (1995). Buyer Characteristics of the Green Consumer and Their Implications for Advertising Strategy. Journal of Advertising, 24, 71-83.

Willer, H., \& Lernoud, J. (2019). The world of organic agriculture. Statistics and emerging trends 2019 (pp. 1-336). Research Institute of Organic Agriculture FiBL and IFOAM Organics International. 
